(C) 2019 IEEE. Personal use of this material is permitted. Permission from IEEE must be obtained for all other uses, in any current or future media, including reprinting/republishing this material for advertising or promotional purposes, creating new collective works, for resale or redistribution to servers or lists, or reuse of any copyrighted component of 1 this work in other works. doi: 10.1109/TSTE.2019.2917831

Publisher version: https://ieeexplore.ieee.org/document/8718333

\title{
Linear and Uniform Swing Dynamics
}

\author{
David Raisz, Senior Member, IEEE, Aysar Musa, Member, IEEE, Ferdinanda Ponci, Senior Member, \\ IEEE, Antonello Monti, Senior Member, IEEE
}

\begin{abstract}
The share of power converter based renewable generation is steadily increasing at the expense of rotating inertia. Virtual Synchronous Machines are deemed to ensure system stability, however, there are no widely accepted criteria for the design of their controllers. In converter-dominated power systems, one of the main challenges will be to analyze power angle stability, because of the large number (and possibly divergent design) of converters. Converters, however, also offer the possibility to shape system dynamics in a way that was impossible with synchronous machines. In this paper we elaborate the new concept of Linearized and Uniform Swing Dynamics. This allows the linearization of the nonlinear swing behavior over almost the entire power range, thereby extending the validity of the small-signal stability analysis techniques for larger disturbances. By properly choosing the controller parameters, the dynamics of the large number of converters expected in the system can be made unified and predictable.
\end{abstract}

Index Terms - Converters, power system control, power system dynamics, power system stability, renewable energy sources.

\section{NOMENCLATURE}

$\begin{array}{ll}\text { Symbol } & \text { Description } \\ \underline{B}, B_{i}, B_{i j} & \text { Susceptance matrix, its } i^{\text {th }} \text { row, its elements } \\ E & \text { Damping constant } \\ E_{k}, E_{p} & \text { Nominal voltage or the ideal remote source } \\ L S D & \text { Kinetic and potential energy components of } T E \\ M & \text { Linear and uniform Swing Dynamics } \\ N L & \text { Noment of inertia } \\ P_{r e f}, P & \text { Power reference, actual electrical power output } \\ R & \text { Real part of the system equivalent impedance } \\ S G & \text { Synchronous generator } \\ S M I B & \text { Single machine infinite bus } \\ S_{S C} & \text { Short circuit power at the grid connection point } \\ T E & \text { Total Energy (Lyapunov function) } \\ V & \text { Terminal voltage of SG or VSM } \\ V S M & \text { Virtual synchronous machine } \\ X & \text { Imaginary part of the equivalent impedance } \\ b & \text { see (17) } \\ \gamma & \text { D/(2M) } \\ \delta & \text { Angle between } E \text { and } V \\ \varepsilon & \text { Voltage tolerance (e.g. } 0.1 \text { for }+/-10 \%) \\ \theta_{V S M} & \text { Phase angle of VSM } \\ \omega, \omega_{s} & \text { Rotational speed, synchronous speed } \\ & \end{array}$

\section{INTRODUCTION}

$\mathrm{T}$ he increasing share of electricity from renewables (mainly wind and solar) leads to a growing number of converters connected to the power system. Scenarios exist for $100 \%$ renewable-based energy supply in Germany by 2050 [1].

Several research efforts therefore aim at the proper establishment of requirements for the design and control of these converters.

Inertia-less system operation is one of the critical challenges for future power systems: disturbances will induce new types of dynamic behavior, which are faster than conventional power system dynamics. These might lead to instability problems and have to be considered in system operation, stability analysis, and control [2], [3].

Moreover, the number of generating units is increasing by several orders of magnitude, as compared to the "conventional" power systems, for which stability concepts are well established. Without a coherent design of converters and their controllers, power system dynamics will be very hard to predict and analyze.

The up-to-date solutions for the inertia problem are Virtual Synchronous Machines (VSM), see [2], [4], [5], [6]. These VSMs are based on the classical representation of the swing equation (SE), which is not the only meaningful option.

With this paper we aim to contribute to the establishment of the requirements for the future, converter-based (VSM-dominated) power systems. Our goal is to tackle the nonlinearity that is inherent to the SE and originating in the power transfer equation. We are doing so by developing the concept of Linear and uniform Swing Dynamics (LSD), which is an improvement of the classical SE-based VSM. It provides: a linear SE dynamics, enhanced system stability and uniform and predictable swing dynamic performance. This concept makes it possible to perform system stability analysis and capture information for the whole state space, i.e. small and larger disturbances, as well. It also provides the same swing-related eigenvalues for almost the whole power range, and therefore it will not be necessary to recalculate the dynamics (eigenvalues, modes etc.) at different operating points.

Power electronic systems provide several degrees of freedom in control system design to implement the LSD concept. However, in this paper we are only assessing a subset of the possible functionalities, namely the voltage-control based LSD.

The disturbances we consider in this paper are the same that are usually considered for small-signal stability analysis [7], e.g. power reference changes or system frequency disturbances, in

\footnotetext{
This work has been funded by the European Commission through the Horizon 2020 research and innovation programme, under the RESERVE project grant agreement No. 727481 .

The authors gratefully acknowledge funding by the German Federal Ministry of Education and Research (BMBF) within the Kopernikus Project ENSURE 'New ENergy grid StructURes for the German Energiewende'.

Authors are with the Institute for Automation of Complex Power Systems, E.ON Energy Research Center, RWTH Aachen University, Mathieustraße 10, 52074 Aachen, Germany. Corresponding Author: David Raisz (draisz@eonerc.rwth-aachen.de), on leave from the Budapest University of Technology and Economics, Budapest, Hungary.
} 
other words: disturbances that do not bring the inverter close to its current-limited mode. We show that by using our approach the linearity assumption holds not only for a small power range around the actual operating point, but over almost the whole power range. We are not focusing on disturbances that are traditionally categorized as large-signal (or transient) stability issues, i.e. faults, because in case of faults the inverters are working in a current-limited mode of operation, to prevent their damage.

We outlined the basic concept of LSD in [10], in which we focused on a single-machine-infinite-bus (SMIB) system, where the grid impedance was represented by a series reactance only. The main contributions of the underlying paper are:

- We provide a Lyapunov stability analysis of the LSD concept

- We derive the concept for a more general class of SMIB systems, where the grid is represented by a resistiveinductive series impedance - mainly typical for distribution systems.

- We extend the concept for multimachine systems and show simulation results.

We note here, that throughout this paper we use two distinct meanings of the word "linearization". On the one hand, it is a mathematical tool for the simplified analysis of an inherently nonlinear phenomenon. On the other hand, as it represents the goal of the LSD concept, it is a special control technology that allows a truly linear physical behavior of a system, which, without LSD, would have behaved in a nonlinear way. The context clearly indicates, which definition we actually mean.

\section{POWER SYSTEM DYNAMIC BEHAVIOR}

In rotor angle stability studies of classical - synchronous generator (SG)-based - power systems, the swing equation (SE) is often used as a low order approximate representation of the electro-mechanical dynamics.

Let us consider a SG connected to a network, represented by its Thévenin equivalent, as shown in Fig. 1. The network equivalent model consists of a constant voltage source $(E)$ and an impedance. Since rotor angle stability analysis is usually performed for transmission systems, the impedance is often approximated by a series reactance only.

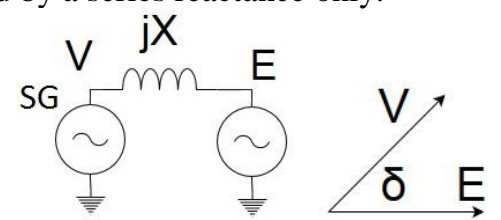

Fig. 1. Simple model for demonstration of the SE

The dynamic behavior of this SMIB system is governed by the following equations [11]:

$$
\begin{gathered}
M \dot{\omega}=P_{r e f}-P-D \dot{\delta} \\
P=\frac{E V}{X} \sin \delta \\
\dot{\delta}=\omega-\omega_{s}
\end{gathered}
$$

with $M$ : inertia constant, $\omega$ : rotational speed, $\omega_{s}$ : synchronous speed, $P_{\text {ref: }}$ reference ("mechanical") power, $P$ : actual power output, $E$ and $X$ : (nominal) voltage and reactance of the equivalent system, $V$ : SG terminal voltage, $\delta$ : angle between $E$ and $V, D$ : damping constant.
The SE is usually linearized with specific assumptions around an operating point to simplify the stability analysis, but then it is applicable only for small disturbances, to capture system stability information for limited operation conditions.

Linearizing (1) - (3) around an operating point (see [11]), we obtain (4) that describes the small-signal behavior of the VSM:

$$
\left[\begin{array}{c}
\Delta \dot{\delta} \\
\Delta \dot{\omega}
\end{array}\right]=\left[\begin{array}{cc}
0 & 1 \\
-\frac{E V}{X M} \cos \delta & -\frac{D}{M}
\end{array}\right]\left[\begin{array}{l}
\Delta \delta \\
\Delta \omega
\end{array}\right]
$$

The eigenvalues of this system can be easily obtained as

$$
-\gamma \pm \sqrt{\gamma^{2}-\frac{E V}{M X} \cos \delta}
$$

with

$$
\gamma=\frac{D}{2 M}
$$

From (2), (4) and (5) it can be observed, that

- the dynamic behavior of this system depends on the actual operating point ( $P$ or $\delta$ ), and

- $\quad$ it has a nonlinear dependence on $\delta$;

- the eigenvalues and the oscillation frequency (if any) can be shaped by controlling $V$.

There are also other possibilities for shaping the system dynamics, but in this paper we will first analyze the most straightforward concept: voltage control. An inverter's voltage control loop can be made significantly faster than excitation control of SGs, which opens new possibilities in designing VSMs.

\section{VIRTUAL SYNCHRONOUS MACHINE (VSM)}

There are several implementation proposals for VSMs in the literature [13].

For the presentation of the LSD concept using time-domain simulations, we will use the so-called "Conceptual Model", which comprises a simple controlled voltage source as VSM.

\section{A. Conceptual Model}

The first model is shown in Fig. 4. The VSM is modeled as a controlled voltage source. Its voltage is controlled by

$$
V(\mathrm{t})=\sqrt{2} V \sin \theta_{V S M}
$$

where $V$ is the desired voltage RMS value, and $\theta_{V S M}$ is obtained from (1) and (3) according to the following control structure:

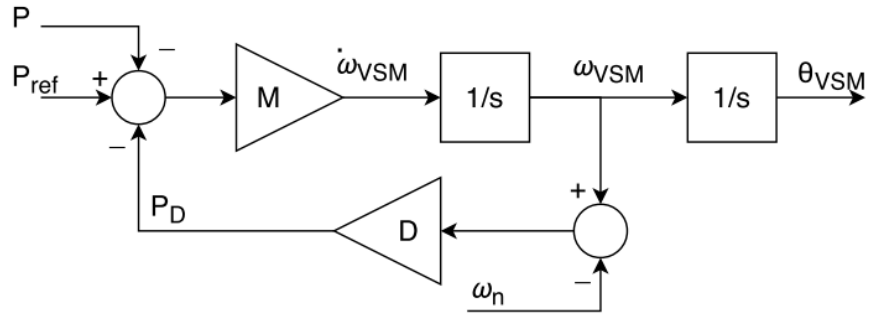

Fig. 2. Control structure underlying the Conceptual Model

Here, $\omega_{n}$ corresponds to the nominal frequency, which is the constant frequency of the voltage source $E$, and $P$ is the actual output active power at the connection point of the VSM. 


\section{B. Cascaded VSM Model}

We also mention here, that in [10] we have used a more detailed model, suitable for more realistic simulations. There we chose a VSM concept that had a cascaded control structure for the following reasons:

- we needed a simple design structure, in which the dynamics of each control loop could be designed fairly independently;

- we found it convenient to have easy access to the voltage reference signal.

Therefore we used the VSM concept presented in [8] and [9]. The configuration is shown in Fig. 3.

We are referring to that model here to show that the LSD concept can be easily embedded into realistic control schemes, in this case for example as an additional block (dashed line) that provides the voltage reference, based on the active power reference. (We have shown in [10] that there is also a possibility to realize LSD behavior by means of a virtual impedance.)

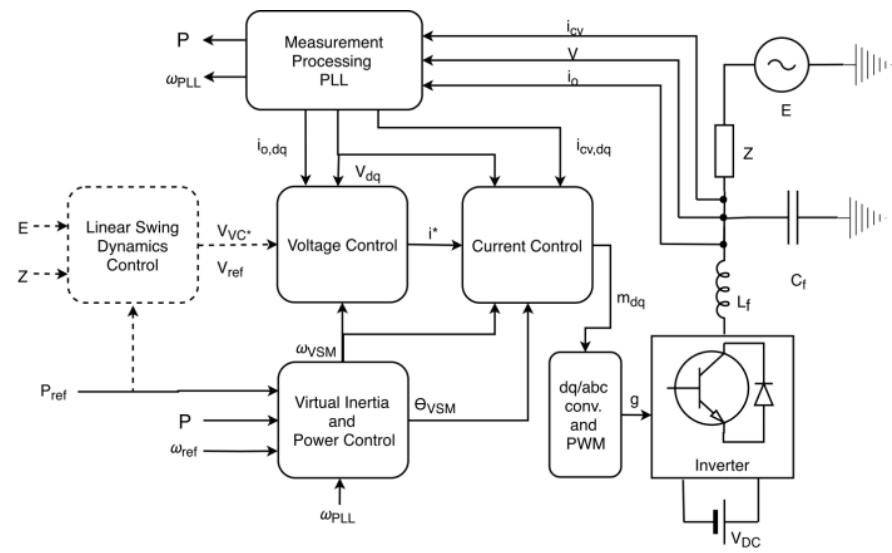

Fig. 3. Structure of the cascaded VSM control concept [10][9]

In this paper, however, we are not focusing on controller implementation and parameter- tuning, but on further development of the concept itself - therefore we will not present here simulation results using the above model.

\section{IMPROVED VSM FOR FUTURE INERTIA-LESS SYSTEMS}

In this Section we will outline the LSD concept, which provides the voltage reference, based on the active power reference.

Throughout the paper we assume, that the dynamic processes shown here can be examined separately from other dynamics, such as PLL or voltage-, current- or DC link control loops. We assume, that the timescales of different control loops are not overlapped.

\section{A. Linear Swing Dynamics (LSD) for the SMIB system}

In order to derive the LSD concept, first we analyze the simplified system shown in Fig. 4.

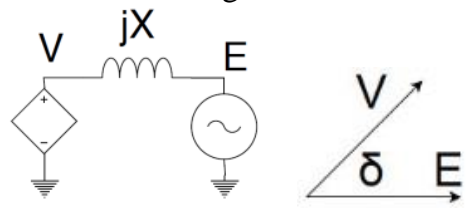

Fig. 4. The inspected system

Our first goal is to linearize (2) with respect to $\delta$, in order to eliminate the dependence of the eigenvalues (5) on the actual operating point or $\delta$.
Since $E$ and $X$ are constant, this linearization can only be done by an appropriate control of $V$ (considering that $V$ usually has to be within a $5 \%$ or $10 \%$ tolerance band around the nominal voltage).

\section{B. Voltage control-based LSD}

The control law can be derived by expressing the expected $P(\delta)$ relationship as in (8) and solving for $V$ :

$$
P=\frac{E V}{X} \sin \delta=\frac{E}{X}(1-\varepsilon) E \delta
$$

where $\varepsilon$ is the voltage tolerance. The solution of (8) will yield

$$
\delta(P)=\frac{P X}{(1-\varepsilon) E^{2}}
$$

and

$$
V(\delta)=\frac{(1-\varepsilon) E}{\sin \delta} \delta
$$

By analyzing the above equations, it can be shown that for $\varepsilon=0.1$ and by using (9) and (10) the $P(\delta)$ relationship will be linear in the angle range $\left[0^{\circ}, 62^{\circ}\right]$, the corresponding power range $[0,0.97]$ and voltage range $[0.9,1.1]$.

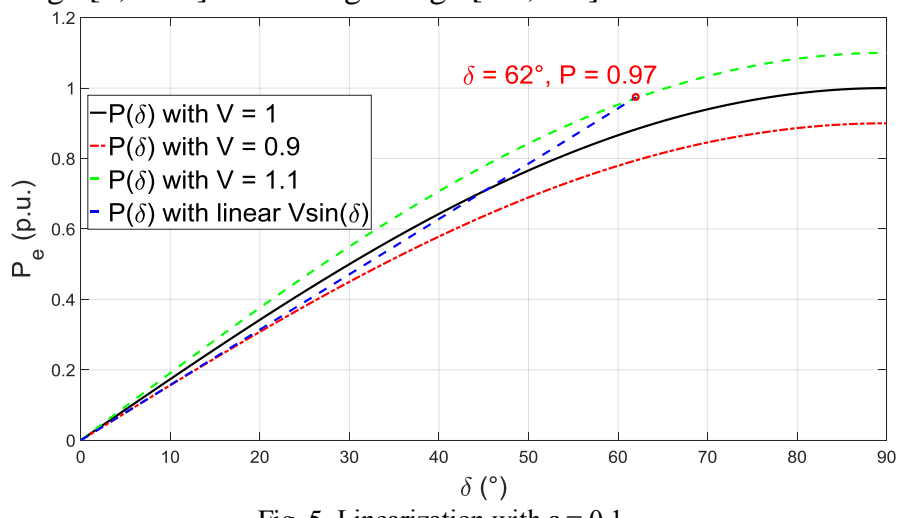

Fig. 5. Linearization with $\varepsilon=0.1$

This means that the power transfer equation can be made linear up to $97 \%$ of the original transfer capacity.

\section{LSD based Improved VSM}

Since (see (8)) $P=\frac{E^{2}}{X}(1-\varepsilon) \delta$, the linearization of $P$ with respect to $\delta$ will yield $\frac{\partial P}{\partial \delta}=\frac{E^{2}}{X}(1-\varepsilon)$.

This means, that by using (9) and (10) the VSM dynamics will be governed by:

$$
\left[\begin{array}{c}
\Delta \dot{\delta} \\
\Delta \dot{\omega}
\end{array}\right]=\left[\begin{array}{cc}
0 & 1 \\
-\frac{(1-\varepsilon) S_{S c}}{M} & -\frac{D}{M}
\end{array}\right]\left[\begin{array}{c}
\Delta \delta \\
\Delta \omega
\end{array}\right]
$$

where $S_{S c}=E^{2} / X$ is the short-circuit power at the grid connection point of the VSM. The eigenvalues of (11) are

$$
-\gamma \pm \sqrt{\gamma^{2}-\frac{(1-\varepsilon) S_{s c}}{M}}
$$

It can be observed from (12) and (6), that the two parameters $M$ and $D$ can be freely chosen, and that these parameters are responsible for shaping the eigenvalues in (12). (Other, e.g. system-level requirements or VSM ratings might pose 
limitations on these parameters, but for the derivation of the concept these constraints are not yet considered.)

One goal in shaping the dynamics could be to achieve uniform eigenvalues and oscillation-free dynamics for all inverters. (The motivation for placing the eigenvalues at certain values can be seen e.g. from [15], where virtual inertia constants $M$ and damping $\mathrm{D}$ - those govern also the eigenvalues - are determined based on required dynamic performance.

Further benefits would be seen in estimating system stability margins and transient behavior of the future power system with a very large number of converter-based generation units). To achieve this, parameters $D$ and $M$ have to fulfil the following conditions:

- $S_{s c} / M$ has to be uniform,

- $\quad \gamma$ has to be uniform (same constant for each inverter)

- and $\gamma^{2} \geq(1-\varepsilon) S_{s c} / M$ for oscillation-free dynamics.

\section{Stability analysis}

Chapter 6.3.2 in [11] presents and analyzes an energy-type Lyapunov function for the system described by (1), (2) and (3). We follow this approach for the system described by (1), (8) and (3). This system has one equilibrium point:

$$
\begin{gathered}
\delta_{s}=\frac{P X}{(1-\varepsilon) E^{2}} \\
\Delta \omega=0
\end{gathered}
$$

Let $T E$ denote the total energy of the system, consisting of the kinetic energy $E_{k}$ and the potential energy $E_{p}$ :

$$
T E=E_{k}+E_{p}
$$

where

$$
E_{k}=\frac{1}{2} M \Delta \omega^{2}
$$

and

$$
E_{p}=\frac{1}{2} b\left(\delta^{2}-\delta_{s}^{2}\right)-P\left(\delta-\delta_{s}\right)
$$

with

$$
b=\frac{E^{2}}{X}(1-\varepsilon)
$$

We now show that the function $T E$ satisfies the definition of a Lyapunov function, i.e. that

(i) it has stationary point at (13)

(ii) it is positive definite in the vicinity of (13)

(iii) its derivative is not positive.

Condition (i) can be checked by calculating

$$
\operatorname{grad} T E=\left[\begin{array}{l}
\frac{\partial T E}{\partial \Delta \omega} \\
\frac{\partial T E}{\partial \delta}
\end{array}\right]=\left[\begin{array}{l}
\frac{\partial E_{k}}{\partial \Delta \omega} \\
\frac{\partial E_{p}}{\partial \delta}
\end{array}\right]=\left[\begin{array}{c}
M \Delta \omega \\
-(P-b \delta)
\end{array}\right]
$$

The gradient is equal to zero at (13).

The second condition can be checked by determining the Hessian matrix given by

$$
H=\left[\begin{array}{cc}
\frac{\partial^{2} T E}{\partial \Delta \omega^{2}} & \frac{\partial^{2} T E}{\partial \Delta \omega \partial \delta} \\
\frac{\partial^{2} T E}{\partial \delta \partial \Delta \omega} & \frac{\partial^{2} T E}{\partial \delta^{2}}
\end{array}\right]=\left[\begin{array}{cc}
M & 0 \\
0 & b
\end{array}\right]
$$

This matrix is always positive definite if $M>0$ and if $b>0$, both of which are always true.

The third condition can be checked by determining

$$
\frac{d T E}{d t}=\frac{d E_{k}}{d t}+\frac{d E_{p}}{d t}
$$

Since (using (1) and (3))

$$
\frac{d E_{k}}{d t}=\left[M \frac{d \Delta \omega}{d t}\right] \Delta \omega=[P-b \delta] \Delta \omega-D \Delta \omega^{2}
$$

and

it follows that

$$
\frac{d E_{p}}{d t}=\frac{\partial E_{p}}{\partial \delta} \frac{d \delta}{d t}=-[P-b \delta] \Delta \omega
$$

$$
\frac{d T E}{d t}=-D \Delta \omega^{2}
$$

and this is always negative.

This means that $\operatorname{TE}(\delta, \Delta \omega)$ is a Lyapunov function, and the equilibrium point (13) is asymptotically stable. (In practice, the voltage magnitude is limited at $1 \pm \varepsilon$, but for short times these limits can be exceeded, as defined e.g. by CBEMA or ITIC curves. These limitations have to be further investigated.)

\section{E. LSD for a network with resistive Thévenin impedance}

In this Section we derive the LSD concept for a more general class of SMIB systems, where the grid is represented by a resistive-inductive series impedance, instead of a pure reactive impedance that is shown in Fig. 4.

Instead of (2), the power transfer across the network equivalent impedance can now be written as

$$
P=\frac{R V(V-E \cos \delta)+E V X \sin \delta}{R^{2}+X^{2}}
$$

In order to be consistent with the pure reactive case, it is straightforward to use the following expressions to define the expected behavior of the linearized system:

$$
\begin{gathered}
V=E(1-\varepsilon) \\
\sin \delta \approx \delta
\end{gathered}
$$

In (8) and (26) we used the first term of the Fourier series of the sine function for linear approximation. However, this approach is not feasible in case of the cosine function. If we use

$$
\cos \delta \approx 1
$$

then we obtain for the linearized $P(\delta)$ relation:

$$
P=\frac{E^{2}(1-\varepsilon)(X \delta-R \varepsilon)}{R^{2}+X^{2}}
$$

For $E=1, R / X=1$ and $|R+j X|=1$ this results in an approximation as shown in an example below: 


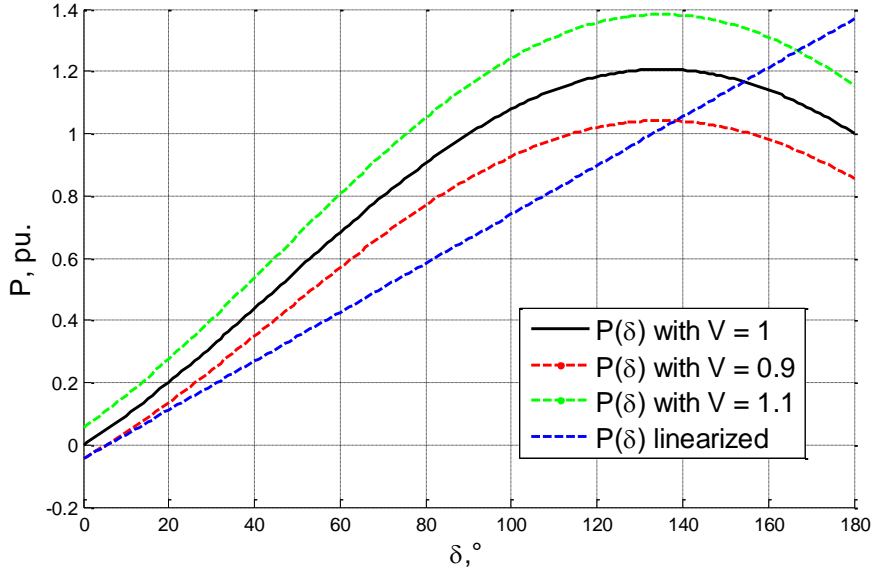

Fig. 6. Linearization for $R / X=1$ and using $\cos \delta \approx 1$

This approximation is inappropriate, since we want the $P(\delta)$ function be a linear relation in between the "lower" und "upper" limit curves determined by $(1-\varepsilon)$ and $(1+\varepsilon)$.

Therefore, we will use the approximation

$$
\cos \delta \approx 1-\frac{\delta}{\pi / 2}
$$

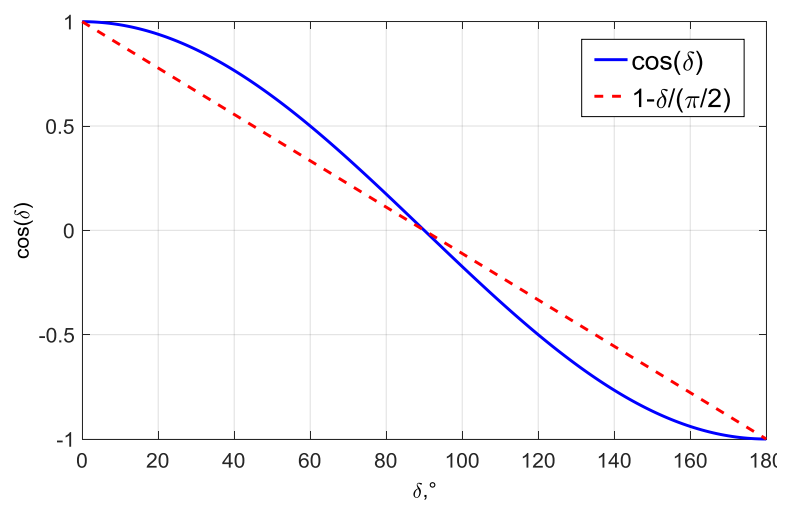

Fig. 7. Linearization concept according to (29)

This way (24) becomes

$$
P=\frac{E^{2}(1-\varepsilon)\left(\frac{2}{\pi} R \delta-R \varepsilon+X \delta\right)}{\left(R^{2}+X^{2}\right)}
$$

For $E=1, R / X=1$ and $|R+j X|=1$ this results in an approximation as shown in an example below:

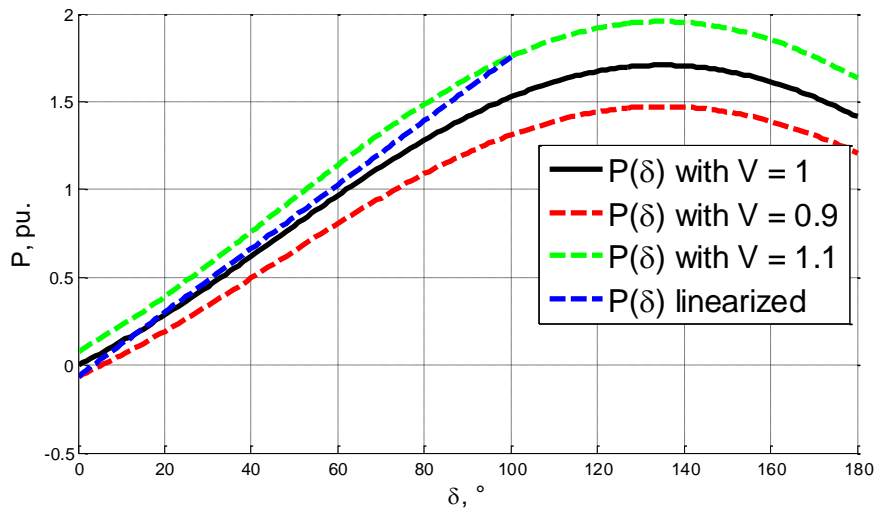

Fig. 8. Linearization for $R / X=1$ and using (29)

For high $R / X$ ratios (above approx. 7) and in a specific power interval, the voltage control will set a voltage reference lower than $(1-\varepsilon)$ pu, as can be seen in Fig. 9 between $\mathrm{P}=1$ and 1.5 .

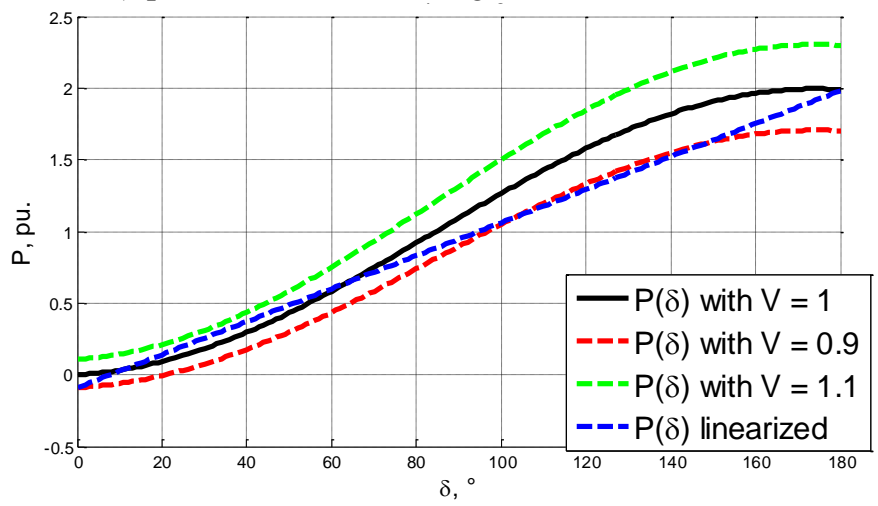

Fig. 9. Linearization for $R / X=10$ and using (29)

In such cases (for $R / X$ ratios above 7), the linear curve can be adjusted. We show in the APPENDIX, that it is possible to find (numerically) a linear curve even for a pure resistive network, as long as $\varepsilon$ is not smaller than $10 \%$. For distribution systems, (where $R / X$ values are significantly above 0 ) usually $10 \%$ voltage tolerance is allowed. (In transmission systems, $\varepsilon=5 \%$ is usually applied, but in these systems $R / X$ values are much smaller than 1, and therefore (9) and (10) can be used.)

For the ease of further analysis, we will assume $R / X<7$ and use (25), (26) and (29).

Solving (29) for $\delta$ we obtain

$$
\delta(P)=\frac{P\left(R^{2}+X^{2}\right)-R E^{2} \varepsilon(1-\varepsilon)}{(1-\varepsilon) E^{2}\left(\frac{2}{\pi} R+X\right)}
$$

and then the solution for $V$ gives:

$$
V(\delta)=E\left(K+\sqrt{K^{2}+(1-\varepsilon)\left(\frac{2 \delta}{\pi}+\frac{\delta}{\rho}-\varepsilon\right)}\right)
$$

with

$$
\mathrm{K}=\frac{1}{2}\left(\cos \delta-\frac{\sin \delta}{\rho}\right)
$$

and

$$
\rho=R / X
$$

(In fact, there are two solutions for $V$, and the above one is the stable solution. The above analytical expressions have been checked with the Symbolic Math Toolbox of Matlab.)

\section{CONVERTER CAPACITY LIMIT}

In this Chapter we will briefly discuss the rated power requirements. Let $\delta_{\max }$ and $P_{\max }$ denote the angle and power at $\frac{\partial \mathrm{P}}{\partial \delta}=0$. For $E=1$ and $|R+j X|=1$ we calculated the apparent power of the inverter for all angles between 0 and $\delta_{\max }$ with different $R / X$ ratios. The required converter capacity (i.e. the maximal apparent power $S_{\text {max }}$ for each R/X ratio) related to $P_{\text {max }}$ is shown in TABLE I.

TABLE I Required inverter oversizing

\begin{tabular}{|c|c|c|c|c|c|c|}
\hline$R / X$ & 0.001 & 0.01 & 0.1 & 1 & 10 & 100 \\
\hline $\begin{array}{c}S_{\max } \\
/ P_{\max }\end{array}$ & 1.25 & 1.24 & 1.18 & 1 & 1 & 1.03 \\
\hline
\end{tabular}


It can be observed that a maximal oversizing of $25 \%$ is sufficient for all conditions, however, at distribution networks with larger $R / X$ ratios practically no oversizing is necessary. It has to be stressed that no further reactive power is required for voltage control purposes, since the voltage control is already taken charge of by the LSD scheme.

\section{MultimachINE SySTEM}

We use a centralized approach to derive the control rules and analyze their behavior in a multimachine system. In this approach, all voltages and angles are assumed to be known, which makes it more suitable for theoretical analysis, than a control method based on local information only.

If branch conductances can be neglected, the active power injections at node $i$ can be expressed as

$$
P_{i}=\sum_{j \neq i} V_{i} V_{j} B_{i j} \sin \left(\delta_{i}-\delta_{j}\right)
$$

where $B_{i j}$ is the respective element of the susceptance matrix $\underline{B}$. Analogously to (9), we seek a voltage control law to achieve

$$
P_{i}=E^{2}(1-\varepsilon) \sum_{j \neq i} B_{i j}\left(\delta_{i}-\delta_{j}\right)
$$

or, equivalently

$$
\underline{P}=-E^{2}(1-\varepsilon) \underline{\underline{B}} \underline{\delta}
$$

where $\underline{P}$ is the column vector of the node injection powers $P_{i}$ and $\underline{\delta}$ is the column vector of the nodal voltage angles $\delta_{i}$.

In order to prove that (36) equals (37), recall that $B_{i i}=$ $-\sum_{j \neq i} B_{i j}$, and therefore, if $B_{i}$ denotes the $i^{\text {th }}$ row of $\underline{\underline{B}}$ :

$$
\begin{aligned}
-B_{i} \underline{\delta}=B_{i}\left[\begin{array}{c}
\vdots \\
\delta_{j} \\
\vdots \\
\delta_{i} \\
\vdots
\end{array}\right] & =-\left(\cdots+B_{i j} \delta_{j}+\cdots+B_{i i} \delta_{i}+\cdots\right) \\
& =-\left(\ldots+B_{i j} \delta_{j}+\cdots-\delta_{i} \sum_{j \neq i} B_{i j}+\cdots\right) \\
& =\sum_{j \neq i} B_{i j}\left(\delta_{i}-\delta_{j}\right)
\end{aligned}
$$

The required angles can now be calculated by solving the linear set of equations (37) for the vector of phase angles. Then the voltage references can be calculated by solving (35) for all voltages. Eq. (35) is nonlinear, and a closed-form solution could not be found for node numbers $N>3$. In such cases, (35) can be solved numerically.

(An analytical solution for $\mathrm{N}=3$ can be found, however, the obtained formulae are too complicated and of little practical relevance, therefore they are not presented here.)

To obtain the eigenvalues of the multimachine LSD-controlled system, we have to derive the expressions $\frac{\partial P_{i}}{\partial \delta_{j}}$ (see again[11], eqs. (12.102) to (12.108)). From (36) it can be seen, that

$$
\frac{\partial P_{i}}{\partial \delta_{j}}=-E^{2}(1-\varepsilon) B_{i j}
$$

Therefore, the elements of the state-space matrix are constant:

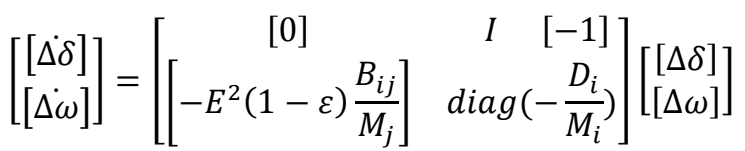

where [.] indicates a matrix structure of appropriate size, composed of the shown elements, $I$ is the identity matrix of appropriate size, and $\operatorname{diag}($.$) indicates a diagonal matrix,$ composed of the shown elements.

This in turn means, that the eigenvalues will be independent of the actual power flows or voltage angles.

Let us consider the following 4-bus system as a demonstration example.

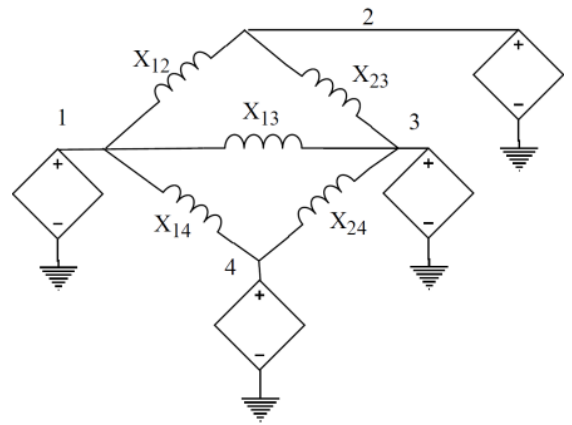

Fig. 10. Four-bus system

The line reactances are assumed to be:

TABLE II Reactance values in the sample system

\begin{tabular}{|l|c|c|c|c|c|}
\hline Pair of nodes (ij) & 12 & 13 & 23 & 14 & 34 \\
\hline$X_{i j}$, pu. & 1 & 1.05 & 0.9 & 1.1 & 1.2 \\
\hline
\end{tabular}

Line resistances are assumed to be zero.

The voltage magnitude at all nodes is kept $1 \mathrm{pu}$. in all cases shown below. We set for all four VSMs the values $M=0.05$ and $D=1$, and we use $E=1$ and $\varepsilon=10 \%$. The system eigenvalues are calculated in four power flow cases (defined by four sets of voltage angles), both without and with LSD.

TABLE III Definition of the cases for eigenvalue calculation: nodal voltage angles

\begin{tabular}{|l|c|c|c|c|c|}
\hline Node $i$ & & 1 & 2 & 3 & 4 \\
\hline \multirow{2}{*}{ Case 1 } & $\delta_{i},{ }^{\circ}$ & 5 & 0 & 5 & 0 \\
\cline { 2 - 6 } & $P_{i}, \mathrm{pu}$. & 0,2 & $-0,2$ & 0,2 & $-0,2$ \\
\hline \multirow{2}{*}{ Case 2 } & $\delta_{i},{ }^{\circ}$ & 60 & 0 & -60 & 0 \\
\cline { 2 - 6 } & $P_{i}, \mathrm{pu}$. & 2,5 & 0,1 & $-2,5$ & $-0,1$ \\
\hline Case 3 & $\delta_{i},{ }^{\circ}$ & 60 & 0 & 60 & 0 \\
\cline { 2 - 6 } & $P_{i}, \mathrm{pu}$. & 1,6 & $-1,8$ & 1,7 & $-1,5$ \\
\hline \multirow{2}{*}{ Case 4 } & $\delta_{i},{ }^{\circ}$ & 90 & 0 & 90 & 0 \\
\cline { 2 - 6 } & $P_{i}, \mathrm{pu}$. & 1,9 & $-2,1$ & 1,9 & $-1,7$ \\
\hline
\end{tabular}

TABLE IV Eigenvalues in the four cases without and with LSD

\begin{tabular}{|c|c|c|c|c|}
\hline Case 1 & Case 2 & Case 3 & Case 4 & LSD (1..4) \\
\hline$-20,00$ & $-20,00$ & $-20,00$ & $-20,00$ & $-20,00$ \\
\hline$-17,92$ & $-19,99$ & $-19,01$ & $-20,00$ & $-18,14$ \\
\hline$-14,95$ & $-19,01$ & $-17,84$ & $-20,00$ & $-15,65$ \\
\hline$-14,79$ & $-17,84$ & $-16,57$ & $-17,89$ & $-15,51$ \\
\hline$-5,21$ & $-2,16$ & $-3,43$ & $-2,11$ & $-4,49$ \\
\hline$-5,05$ & $-0,99$ & $-2,16$ & 0,00 & $-4,35$ \\
\hline$-2,08$ & $-0,01$ & $-0,99$ & 0,00 & $-1,86$ \\
\hline
\end{tabular}

In all four test cases the eigenvalues with LSD remain the same, as seen in the last column of TABLE IV. 


\section{DYNAMIC SIMULATION AND RESULTS}

In this Section we present time domain simulation results to underpin the theoretical analysis shown in previous chapters.

\section{A. SMIB - Conceptual model}

First, we present simulation results obtained with the Conceptual Model. We use $E=231 \mathrm{~V}, X=0.19 \Omega, M=2000$ and $D=40000(R=0.19 \Omega$, where applicable $)$. In all our simulations, the nominal frequency is $50 \mathrm{~Hz}$. The $P(\delta)$ curves have been obtained by stepping $P_{\text {ref }}$ from 0.1 to 0.5 and finally to 0.9 fraction of the power $P=E^{2} / X=276 \mathrm{~kW}$. The simulation results with a pure inductive equivalent network can be seen in Fig. 11, without LSD (left side), and with LSD (right side).
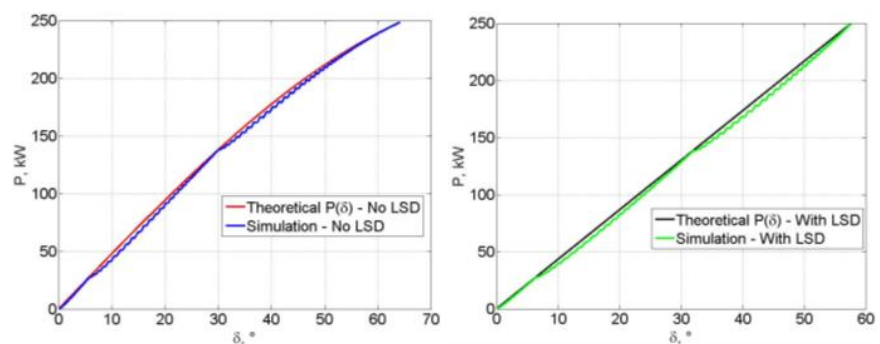

Fig. 11. Simulation results with the Conceptual Model, pure inductive grid

Results with a pure resistive equivalent network can be seen in Fig. 12, without LSD (left side), and with LSD (right side).
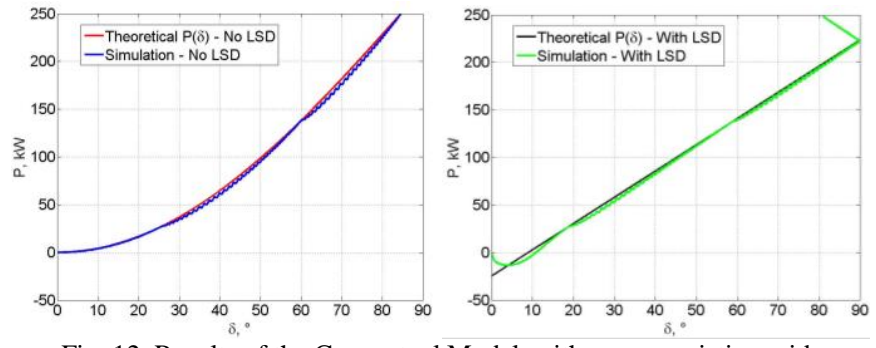

Fig. 12. Results of the Conceptual Model, with a pure resistive grid

It can be observed, that the steady-state $P(\delta)$ value pairs are found at the theoretically determined (sinusoidal and LSDlinearized) curves, and these curves are closely followed during transients, too.

\section{B. Multimachine system}

For the simulation of the LSD concept applied to a multimachine system, we used the same four-bus system as shown in Fig. 10. The active power references were set to the values shown in TABLE $\mathrm{V}$.

TABLE V Active power reference values for the 4-bus system

\begin{tabular}{|l|c|c|c|c|}
\hline pu. & $P_{\text {ref }, 1}$ & $P_{\text {ref }, 2}$ & $P_{\text {ref }, 3}$ & $P_{\text {ref }, 4}$ \\
\hline $\mathrm{t}=0 \mathrm{~s}$ & 1.9 & -2 & 1.95 & -1.85 \\
\hline $\mathrm{t}=5 \mathrm{~s}$ & 1.5 & -1 & 1.2 & -1.7 \\
\hline $\mathrm{t}=10 \mathrm{~s}$ & 1 & -0.5 & 0.5 & -1 \\
\hline
\end{tabular}

The voltage references were calculated periodically (at every $10 \mathrm{~ms}$ ) by first solving (37) for $\underline{\delta}$ (we will denote the resulting vector of time functions $\underline{\delta}_{\operatorname{sim}}(t)$ later) and then solving (35) for $\underline{V}$ numerically. To prevent numerical instabilities, $P_{\text {ref }, i}(t)$ values were used in these equations for $t<3 \mathrm{~s}$, instead of the actual output powers $P_{i}(t)$, which were used after $t=3 \mathrm{~s}$.

The reference and actual power outputs are presented in Fig. 13.

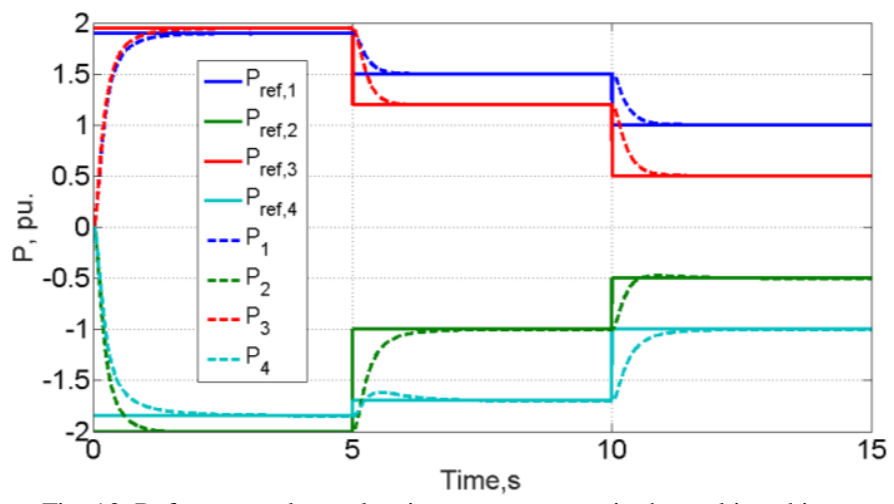

Fig. 13. Reference and actual active power outputs in the multimachine system

In order to prove the linear $\underline{P}(\delta)$ behavior, we construct

$$
\underline{P}_{c a l c}(t)=-E^{2}(1-\varepsilon) \underline{\underline{B}} \underline{\delta}_{s i m}(t)
$$

This is the ideal, expected linear relationship between the angles and the nodal powers. In Fig. 14 we present that the error between the ideal active powers $\underline{P}_{\text {calc }}(t)$ and the simulated output powers $\underline{P}(t)$ is sufficiently small. We construct a measure for nonlinearity as follows:

$$
N L_{i}(t)=\frac{P_{\text {calc }, i}(t)-P_{i}(t)}{P_{i}(t)}
$$

It can be observed that $N L_{i}(t)$ is close to zero in steady state, and below 2\% during transients (except for the first $3 \mathrm{~s}$ period, which we explained earlier).

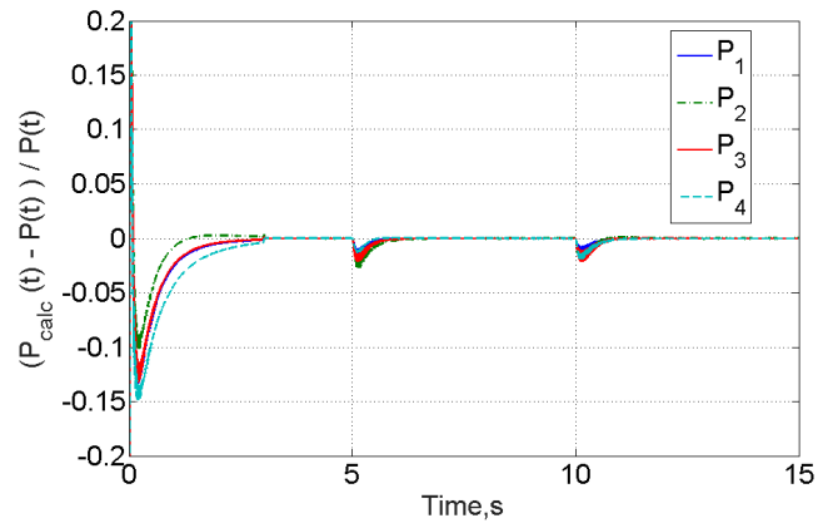

Fig. 14. Measure of $\underline{P}(\delta)$ nonlinearity in the multimachine system

\section{CONCLUSIONS}

In this paper we further developed the LSD concept, which was first outlined in [10]. We have shown, that it is applicable not only to pure inductive equivalent networks, but also inductiveresistive (and also pure resistive) SMIB networks, which are typical representations of distribution systems.

We have shown, that it is possible to control the voltage of VSMs in a way that the $P(\delta)$ relation becomes a linear function of the power angle. This in turn means that the small-signal dynamics can be made independent on the actual operating point. We have also demonstrated, that this is possible for multimachine systems, too. We claim that for systems with high VSM penetration, this concept makes it possible to perform system stability analysis and capture information for the whole 
power range, i.e. small and large disturbances (that do not bring the converter into current-limited mode).

\section{FUTURE WORK}

Several issues have to be clarified regarding the theory and practical applicability of the LSD concept. Some of these are shortly discussed here, though they do not constitute the main contributions of this paper.

\section{A. Controller design}

When implementing LSD in a real inverter control structure (e.g. the one in Fig. 3), particular attention has to be paid to parameter tuning. The VSM dynamics should be decoupled from other control loops, their bandwidth should not be overlapped. This might be a challenge in case of some PLL methods. However - since this is a general problem for VSCs that provide services to the grid - there is significant ongoing research effort in developing fast, linear and accurate synchronization/frequency tracking solutions [14]. Another direction of further investigations is the analysis of different VSM structures for which the controller tuning process might be less challenging (e.g. PLL-less Synchronverters [12]).

\section{B. DC voltage control}

So far we have assumed a constant DC-link voltage. In a realcase application the back-end subsystem will be some power source, and in this case the DC voltage dynamics should also be considered and the control loops complemented accordingly.

\section{Voltage stability}

Since the presented LSD scheme is integrating voltage control, it has to be investigated, how voltage stability will be affected. We performed a network feasibility assessment for the system shown in Fig. 15.

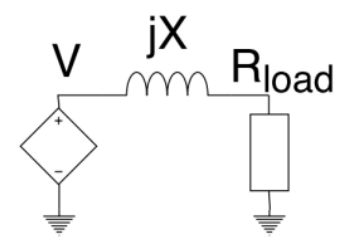

Fig. 15. Assessment of network feasibility

We assumed $X=1$ and the load resistance $R_{\text {load }}$ was varied between 0.1 and 20. For the LSD algorithm, $E=1$ was assumed, and for the sake of comparability with the non-LSD case, $V=1$ was set. "

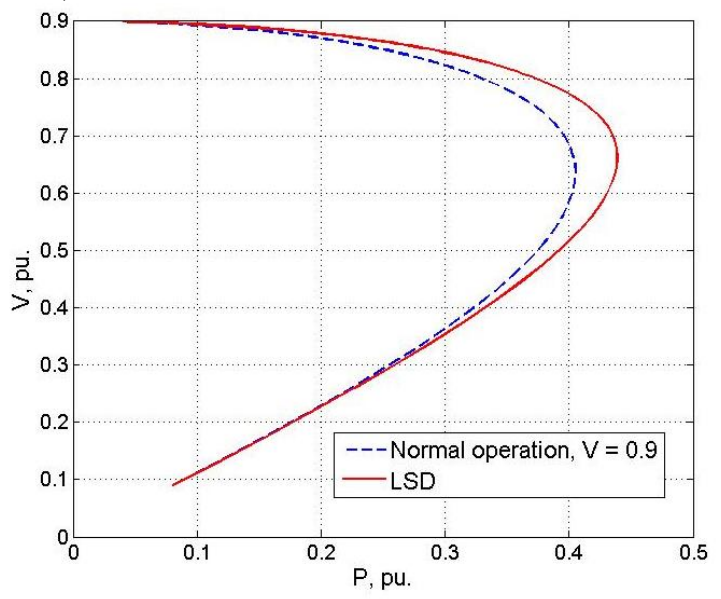

Fig. 16. Voltage stability assessment
It can be observed, that by using LSD the stable region was extended, i.e. larger loads can be supplied without voltage collapse. A complete proof and dynamic investigations will be reported in subsequent publications.

\section{Other topics}

It was shown (see Fig. 5) that LSD can linearize the power transfer behavior and thus the dynamics, up to $97 \%$ of the maximum non-LSD transfer capacity (in a pure reactive network). It has to be investigated how the system will behave in case when this limit is passed. The voltage cannot be increased further (though this might be allowed for a limited time interval), and the shift between different control modes might pose a challenge.

Also the behavior under fault conditions has to be defined and analyzed.

Further research is needed for the extension of the method to multimachine systems with resistive-inductive impedances, and also to handle negative relative angles.

Finally, a decentralized approach has to be developed, where the local control parameters are determined based on locally available measurements only (e.g. by using some equivalent dynamic model of the network - as seen from an inverter connection point). This is relevant from the practical applicability point of view.

\section{APPENDIX}

In this Section we will show that for all values of $R / X$ and $\varepsilon=10 \%$ there exists a linear $L(\delta, m)$ curve as well as $m_{1}, m_{2}$, $\delta_{1}$ and $\delta_{2}$ positive scalars with the following properties:

$$
\begin{gathered}
L(\delta, m)=P_{\text {lower }}(0)+m \delta \\
L\left(\delta_{1}, m_{1}\right)=P_{\text {upper }}\left(\delta_{1}\right) \\
L\left(\delta_{2}, m_{2}\right)=P_{\text {lower }}\left(\delta_{2}\right) \\
\frac{\partial P_{\text {upper }}}{\partial \delta}\left(\delta_{1}\right)=m_{1} \\
\frac{\partial P_{\text {lower }}}{\partial \delta}\left(\delta_{2}\right)=m_{2} \\
m_{1} \geq m_{2} \\
\delta_{2} \geq \delta_{1}>0
\end{gathered}
$$

where

$$
\begin{aligned}
& P_{\text {lower }}=P \text { with } V=E(1-\varepsilon) \text { in }(24) \\
& P_{\text {upper }}=P \text { with } V=E(1+\varepsilon) \text { in }(24)
\end{aligned}
$$

In other words, we will show that there exists a linear curve $(L)$ in between two tangents to the two tolerance curves $P_{\text {lower }}(\delta)$ and $P_{\text {upper }}(\delta)$, with a starting point at $P_{\text {lower }}(0)$. This is illustrated for $R=1, X=0$ in Fig. 17.

From (24) we can obtain

$$
\frac{\partial P}{\partial \delta}=\frac{E V(X \cos \delta+R \sin \delta)}{R^{2}+X^{2}}
$$

Substituting (44) and (46) into (43), and using (46), we obtain

$$
P_{\text {upper }}\left(\delta_{1}\right)=P_{\text {lower }}(0)+\delta_{1} \frac{\partial P_{\text {upper }}}{\partial \delta}\left(\delta_{1}\right)
$$

We can solve this numerically for $\delta_{1}$, by using (24) and $V=$ $E(1+\varepsilon)$. From $\delta_{1}$ we can determine $m_{1}$ using (46).

Similarly, we can obtain $\delta_{2}$ and $m_{2}$ using (24), (45), (47) and $V=E(1-\varepsilon)$. 


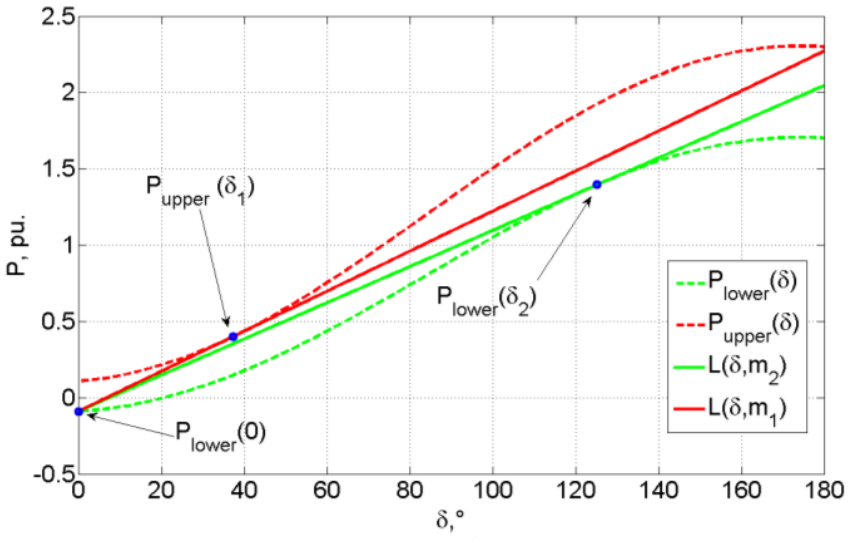

Fig. 17. Illustration of eq. (35) - (49)

In TABLE VI we show exemplary results for $E=1, \varepsilon=10 \%$ and $|R+j X|=1$.

TABLE VI Slopes of the tangents to the $P(\delta)$ curves

\begin{tabular}{|c|c|c|}
\hline$R / X$ & $m_{1}$ & $m_{2}$ \\
\hline 10 & 0.7514 & 0.6796 \\
\hline 50 & 0.6730 & 0.6577 \\
\hline infinite & 0.6526 & 0.6522 \\
\hline
\end{tabular}

Since in all cases (48) holds, we can conclude, that in all cases it is possible to find a linear curve between $P_{\text {lower }}(\delta)$ and $P_{\text {upper }}(\delta)$, with a starting point at $P_{\text {lower }}(0)$. Its slope can be chosen between $m_{1}$ and $m_{2}$.

\section{REFERENCES}

[1] Energy Concept 2050 for Germany with a European and Global Perspective, June 2010, http://www.fvee.de/fileadmin/publikationen/ Politische_Papiere_FVEE/10.06.Energy_Concept_2050/EK2010_EN.pdf

[2] H. Bevrani,"Robust Power System Frequency Control", 2nd Edition, Springer International Publishing Switzerland, 2014.

[3] A. Benchaib,"Advanced Control of AC/DC Power Networks, system of systems approach based on spatio-temporal scales", Wiley, USA, 2015.

[4] J. Zhou and Y. Ohsawa, Improved swing equation and its properties in synchronous generators, Circuits and Systems I: Regular Papers, IEEE Tr. on Circuits and Systems, vol. 56, no. 1, pp. 200-209, Jan 2009.

[5] K. Visscher and S. W. H. De Haan, "Virtual synchronous machines (VSG's) for frequency stabilisation in future grids with a significant share of decentralized generation," CIRED Seminar 2008: SmartGrids for Distribution, Frankfurt, 2008, pp. 1-4.

[6] Q. C. Zhong, "Virtual Synchronous Machines: A unified interface for grid integration," in IEEE Power El. Mag., vol. 3, no. 4, pp. 18-27, Dec. 2016.

[7] P. Kundur et al., "Definition and classification of power system stability IEEE/CIGRE joint task force on stability terms and definitions," in IEEE Transactions on Power Systems, vol. 19, no. 3, pp. 1387-1401, Aug. 2004.

[8] S. D'Arco, J. A. Suul and O. B. Fosso, "Control system tuning and stability analysis of Virtual Synchronous Machines," 2013 IEEE Energy Conversion Congress and Exposition, Denver, CO, 2013, pp. 2664-2671.

[9] S. D'Arco, J. A. Suul and O. B. Fosso, "Small-signal modelling and parametric sensitivity of a Virtual Synchronous Machine," PSCC 2014

[10] David Raisz, Aysar Musa, Ferdinanda Ponci, Antonello Monti, "Linear and Uniform System Dynamics of Future Converter-Based Power Systems", accepted for presentation at the IEEE PES General Meeting 2018, 5-9 August, 2018, Portland, Oregon, USA

[11] Jan Machowski, Janusz Bialek, Dr. Jim Bumby: „Power System Dynamics: Stability and Control",Wiley,2008, ISBN:978-0-470-72558-0

[12] Q. C. Zhong, P. L. Nguyen, Z. Ma and W. Sheng, "Self-Synchronized Synchronverters: Inverters Without a Dedicated Synchronization Unit," in IEEE Transactions on Power Electronics, vol. 29, no. 2, pp. 617-630, Feb. 2014. doi: 10.1109/TPEL.2013.2258684

[13] S. D'Arco and J. A. Suul, "Virtual synchronous machines Classification of implementations and analysis of equivalence to droop controllers for microgrids," 2013 IEEE Grenoble Conference, Grenoble,
2013, pp. 1-7. doi: 10.1109/PTC.2013.6652456

[14] Á. Ortega and F. Milano, "Comparison of different PLL implementations for frequency estimation and control," 18th International Conference on Harmonics and Quality of Power (ICHQP), Ljubljana, 2018

[15] Placement and Implementation of Grid-Forming and Grid-Following Virtual Inertia and Fast Frequency Response," to appear in IEEE Transactions on Power Systems. doi: 10.1109/TPWRS.2019.2892290

\section{BIOGRAPHIES}

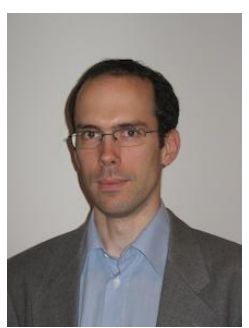

David Raisz (M'06-SM'18) received his M.Sc degree and his $\mathrm{PhD}$ in Electrical Engineering from Budapest University of Technology and Economics (BUTE), Budapest, Hungary, in 2000 and 2011 respectively. From 1999 to 2001 he joined Graz University of Technology, Austria, as a guest researcher. From 2012 until 2016 he led the Power Systems and Environment Group at the Dept. of Electric Power Engineering at BUTE, as Associate Professor. In 2017 he joined the Institute for Automation of Complex Power Systems within the E.ON Energy Research Center at RWTH Aachen University. He has been working on or leading more than 40 industrial and research projects.

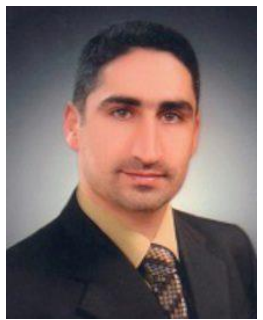

Aysar Musa received the B.Sc. degree in power engineering from University of Mosul, Mosul, Iraq, in 2004, and the M.Sc degree in electrical and electronic engineering from University of Eastern Mediterranean, Famagusta, North Cyprus, in 2013. He is currently working toward the Ph.D. degree at the Institute for Automation of Complex Power Systems,E.ON Energy Research Center, RWTH Aachen University, Aachen, Germany. From 2005 to 2011, he was an Operating System Engineer in the Northern Transmission Electrical Networks, Ministry of Electricity, Iraq. In 2013, he became a Senior Power Systems Engineer. Since 2014, he has been a Research Associate with the Institute for Automation of Complex Power Systems, E.ON Energy Research Center, RWTH Aachen University. His research interests include renewable energy integration, as well as control and stability of low-inertia power systems and hybrid AC/DC networks.

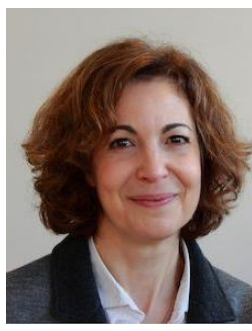

Ferdinanda Ponci (M'00-SM'08) received thePh.D. degree in electrical engineering from thePolitecnico di Milano, Milan, Italy, in 2002. She joined the Department of Electrical Engineering, University of South Carolina, Columbia, SC,USA, as an Assistant Professor in 2003, and became an Associate Professor in 2008. In 2009, she joined the Institute for Automation of Complex Power Systems, RWTH Aachen University, Aachen, Germany, where she is currently a Professor of monitoring and distributed control for power systems. She is aSenior Member of the AdCom of the IEEE Instrumentation and Measurement Society.

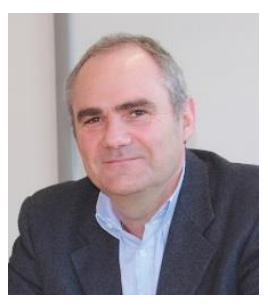

Antonello Monti (SM'2002) received his M.Sc degree (summa cum laude) and his PhD in Electrical Engineering from Politecnico di Milano, Italy in 1989 and 1994 respectively. He started his career in Ansaldo Industria and then moved in 1995 to Politecnico di Milano as Assistant Professor. In 2000 he joined the Department of Electrical Engineering of the University of South Carolina (USA) as Associate and then Full Professor. Since 2008 he is the director of the Institute for Automation of Complex Power System within the E.ON Energy Research Center at RWTH Aachen University. Dr. Monti is author or co-author of more than 300 peer-reviewed papers published in international Journals and in the proceedings of International conferences. He is a Senior Member of IEEE, Associate Editor of the IEEE System Journal, Associate Editor of IEEE Electrification Magazine, Member of the Editorial Board of the Elsevier Journal and Sustainable Energy, Grids and Networks, and member of the founding board of the Springler Journal Energy Informatics. Dr. Monti is the recipient of the 2017 IEEE Innovation in Societal Infrastructure Award. 